\title{
Nonequilibrium phase transitions in a model for the origin of life
}

\author{
Claudia P. Ferreira and J. F. Fontanari \\ Instituto de Física de São Carlos \\ Universidade de São Paulo \\ Caixa Postal 369 \\ 13560-970 São Carlos SP, Brazil
}

\begin{abstract}
The requisites for the persistence of small colonies of self-replicating molecules living in a two-dimensional lattice are investigated analytically in the infinite diffusion or mean-field limit and through Monte Carlo simulations in the position-fixed or contact process limit. The molecules are modeled by hypercyclic replicators $A$ which are capable of replicating via binary fission $A+E \rightarrow 2 A$ with production rate $s$ as well as via catalytically assisted replication $2 A+E \rightarrow 3 A$ with rate $c$. In addition, a molecule can degrade into its source materials $E$ with rate $\gamma$. In the asymptotic regime the system can be characterized by the presence (active phase) and the absence (empty phase) of replicators in the lattice. In both diffusion regimes, we find that for small values of the ratio $c / \gamma$ these phases are separated by a second-order phase transition which is in the universality class of the directed percolation, while for small values of $s / \gamma$ the phase transition is of first order. Furthermore, we show the suitability of the dynamic Monte Carlo method, which is based on the analysis of the spreading behavior of a few active cells in the center of an otherwise infinite empty lattice, to address the problem of the emergence of replicators. Rather surprisingly, we show that this method allows an unambiguous identification of the order of the nonequilibrium phase transition.
\end{abstract}

PACS: $87.10+\mathrm{e}, 87.90 .+\mathrm{y}, 89.90+\mathrm{n}$ 


\section{Introduction}

The most fundamental event in the history of life was probably the appearance via spontaneous creation of a molecule capable of replicating itself (replicator). Given a possible mechanism of replication, which in this case is some form of template activity, the evolution of such replicators has been extensively investigated through the chemical kinetics formalism put forward by Eigen and co-workers in the seventies 11. 2]. Those studies have raised a series of objections to the simplistic view of the emergence of a complex organism from a collection of competing species of replicators. For instance, the finding that the length of a molecule (polynucleotide) is limited due to the finite replication accuracy per nucleotide has prompted the proposal of models that incorporated some sort of cooperation between the replicators, such as the molecular catalytic feedback networks termed hypercycles [2]. These models, however, have attracted their own criticisms since, as pointed out by Maynard Smith, giving catalytic support in such molecular networks is in fact an altruistic behavior and so they are extremely vulnerable to the presence of parasites, i.e., molecules that do not reciprocate the catalytic support they receive [3]. A possible solution to this problem is provided by the structured deme formulation of group selection [4], where it is assumed that the replicators are spatially localized, say, in rock crevices or water droplets, so that the benefits accrued from cooperation are directed mostly to the members of the catalytic network [5, 6, [7. Yet another successful approach to the problem of resistance against parasites is based on a reaction-diffusion system where replication and diffusion taking place on an adsorbing surface generate self-organized spiral structures 8, 9]. Interestingly, as these spatial structures, which greatly increase the stability of the hypercycles against parasites, can be viewed as super-organisms that approach is also related to the group selection theory $\mathbb{8}$.

Since in the prebiotic or chemical evolution context, natural selection is essentially the dynamics of replicators, it is not surprising that most of the studies in this subject have focused almost exclusively on the competition between replicators, among which the so-called malthusian and hypercyclic replicators are the most important [2, 5]. The former corresponds to the simplest reproduction process, namely, the binary fission of a parent replicator and is modelled by the chemical reaction

$$
A+E \stackrel{s}{\rightarrow} 2 A
$$

where $A$ is the replicator and $E$ is the source materials (mononucleotide resources). It is well-known that the concentration of $A$ grows exponentially with the rate constant $s$, provided that the concentration of $E$ is kept constant, hence the name malthusian replicator. To avoid this explosive growth, one usually imposes a constraint on the total concentration of replicators which can be implemented in practice by a dilution flux [1]. Alternatively, one can allow the replicators to be degraded by hydrolysis into its mononucleotide components $E$ according to the reaction

$$
A \stackrel{\gamma}{\rightarrow} E
$$

which seems a more natural approach to limit the growth of $A$. 
As best exemplified by sexual reproduction, there are situations that cannot be described by (1) since two replicators are necessary to produce a third one. In this case the corresponding chemical reaction is

$$
2 A+E \stackrel{c}{\rightarrow} 3 A
$$

which leads to a hyperbolic growth of the concentration of replicator $A$ [2]. A hypercyclic replicator is defined as one that can replicate itself using both (11) and (3) reaction schemes. Actually, the term hypercycle derives from the superimposition of the catalytic replication cycle (3) on the self-replication cycle (11). Of course, the limit $c=0$ corresponds to the malthusian replicator while $s=0$ can be associated to an obligatory sexual replicator.

In contrast to previous works that have concentrated on the competition between replicators either of the same kind but with different production rates [1], 2] or of different kinds [5], in this paper we address a more fundamental problem that has received comparatively little attention, namely, the stability of the different kinds of replicators, viewed here as an active (ordered) phase of the molecular system, against the empty (disordered) phase composed of the resource materials only. This lack of interest was probably due to the fact that the usual kinetics formalism used to study the dynamics of replicators does not represent the mononucleotide resource dynamics explicitly (see however 10, 11), thus precluding the study of the issues addressed in the present contribution. More pointedly, we consider the dynamics of a population of identical hypercyclic replicators on a lattice space both in the deterministic infinite diffusion (mean-field) limit and in the stochastic position-fixed (contact process) limit where each replicator on a lattice cell never moves. The last limit is particularly interesting because it allows the connection between the replicator models and some standard models of nonequilibrium phase transition in a lattice (e.g. directed percolation) 12, 13, 14. As a result, the powerful analytical tools of statistical mechanics can be readily used to advance our understanding of the evolution of replicators. Of particular relevance is the so-called dynamic Monte Carlo method whose idea is to set the system initially in the empty state with a seed of replicators in the center of the lattice and then study the subsequent spreading of activity [12, 13, 14]. More importantly, the thorough analysis of both limits exposes the limitations of the widely used deterministic chemical kinetics or mean-field formalism to study the problem of the emergence of life.

The remainder of the paper is organized as follows. In Sec. 2 we present the set of rules that govern the evolution of a population of hypercyclic replicators in a two-dimensional square lattice. The mean-field or infinite diffusion limit, which models an ideally mixed medium, is studied analytically in Sec. 3. The results are summarized in a phase diagram showing the regions of stability of the empty and active regimes in the space of the control parameters of the model. Those regions are delimitated by continuous as well as discontinuous transition lines that join at a tricritical point. In Sec. 1 we study the position-fixed or contact process limit using mainly the dynamic Monte Carlo method which allows the computation of the critical dynamic exponents that describe quantitatively the spreading of a vanishingly small population of replicators. Finally, some concluding remarks are 
presented in Sec. 5. In particular, we compare the hypercyclic replicator model with Schlögl's models of nonequilibrium phase transition in reaction-diffusion systems [15.

\section{The model}

It has been suggested that chemical evolution started with a surface-bonded autocatalytic chemical network as there are enormous thermodynamics and kinetics advantages of surface binding reactions, especially in the case of reactions that require unlikely collisions of many reactants $[16]$. The binding must be strong enough to keep the reactants on the surface but also flexible enough to allow their slow migration on it. Interestingly, if this proposal proves correct it will probably lead to the replacement of the popular notion of a primitive soup by that of a primitive pizza instead [17. Accordingly, we define our replicator model in a two-dimensional space consisting of $L \times L$ cells in a square toroidal lattice. Each cell is either empty or occupied by a replicator and it is assumed that an empty cell contains all source material required to assemble a new replicator. The evolution of the population of replicators is governed by the following local rules:

(1) A replicator has a probability $\gamma$ of decaying; after decay the cell becomes empty. This rule is motivated by the hydrolysis reaction (2).

(2) A replicator in one of the four first neighbor cells (von Neumann neighborhood) of an empty cell can replicate into that cell with probability $s$. This process is referred to as non-catalised self-replication and is motivated by the reaction (11).

(3) Regardless of the previous rule, a replicator in the von Neumann neighborhood of an empty cell can replicate into that cell if there are other replicators in the intersection of the Moore neighborhoods of both cells. The probability of this type of replication, which is motivated by reaction (3), is $c$ for each pair of replicators. We recall that the Moore neighborhod of a given cell consists of its first and second nearest neighbors, adding up to eight cells.

Hence in the extreme situation where an empty cell is surrounded by eight replicators, it can become occupied with probability $4 s+16 c$. To carry out the simulations we choose the parameters $c$ and $s$ such that $4 s+16 c \leq 1$. These rules are applied simultaneously to all cells in the lattice so our model can be viewed as a two-dimensional stochastic cellular automaton. Actually the model is essentially an adaptation to one-membered hypercycles of the spatial cellular automaton model of multi-membered hypercycles proposed by Boerlijst and Hogeweg [8]. The dynamics defined by the rules given above is manifestly irreversible and, in particular, the state characterized by empty cells only is an absorbing state, i.e., a configuration from which the system cannot escape. In this sense, the principle of detailed balance is broken and the active stationary state is in fact in nonequilibrium. Although the more realistic situation is a diffusion-controled reaction where each reactant can 
move randomly on the lattice, in this paper we choose to study in detail the simpler extreme cases of infinite diffusion (mean field) and no diffusion (contact process). Of course, we hope that features common to both limits will be present in the finite diffusion situation as well.

\section{The mean-field limit}

The mean-field limit describes exactly an infinite population of reactants in an ideally mixed medium and so it is equivalent to the usual chemical kinetics formulation. Neglecting spatial correlations among cells, i.e., assuming that at any time the molecules are distributed randomly over the lattice cells, it is straightforward to write the evolution equation for the density of replicators or occupied cells at time $t$, namely,

$$
\rho_{t+1}=\rho_{t}(1-\gamma)+4 \rho_{t}\left(1-\rho_{t}\right)\left(s+4 c \rho_{t}\right) .
$$

We will consider only the stationary solutions $\rho_{t+1}=\rho_{t}=\rho$ of this equation. The absorbing (empty) state $\rho=0$ is always a solution, while the non-zero solutions are given by the roots of the quadratic equation

$$
\tilde{c} \rho^{2}-\rho(\tilde{c}-\tilde{s})+1-\tilde{s}=0
$$

where we have introduced the dimensionless parameters

$$
\tilde{c}=\frac{16 c}{\gamma} \quad \text { and } \quad \tilde{s}=\frac{4 s}{\gamma} .
$$

This equation has real roots provided that the condition $(\tilde{c}+\tilde{s})^{2} \geq 4 \tilde{c}$ is satisfied. In addition we can easily show that: (i) for $\tilde{s}<1$ and $\tilde{c}>\tilde{s}$ both roots are positive; (ii) for $\tilde{s}<1$ and $\tilde{c}<\tilde{s}$ both roots are negative; and (iii) for $\tilde{s}>1$ only one of the roots is negative. Furthermore, though the solution $\rho=0$ exists in the entire plane $(\tilde{c}, \tilde{s})$, it is stable only if the condition

$$
\left.\frac{\partial \rho_{t+1}}{\partial \rho_{t}}\right|_{\rho=0}=1-\gamma+4 s<1,
$$

which reduces to $\tilde{s}<1$, is satisfied. In the region where the non-zero roots are physical (i.e., real and positive), the stable root is always the largest one. In Fig. 1 we show the steady-state density of replicators for two different choices of initial density. We identify three distinct phases: the absorbing or empty phase $(E)$ associated to the solution $\rho=0$; the replicating or active phase $(A)$ associated to the solution $\rho>0$; and the phase labeled $(E A)$ where both solutions $\rho=0$ and $\rho>0$ are stable. In this phase, the outcome of the dynamics is not determined by the control parameters only but also by the initial abundance of replicators. ¿From this figure it is clear that the system undergoes a continuous non-equilibrium phase transition from phase $(E)$ to phase $(A)$ at $\tilde{s}=\tilde{s}_{c}=1$ and $\tilde{c}<1$. Explicitly, near this transition the density of replicators can be written as

$$
\rho \approx \frac{\tilde{s}-1}{1-\tilde{c}} .
$$



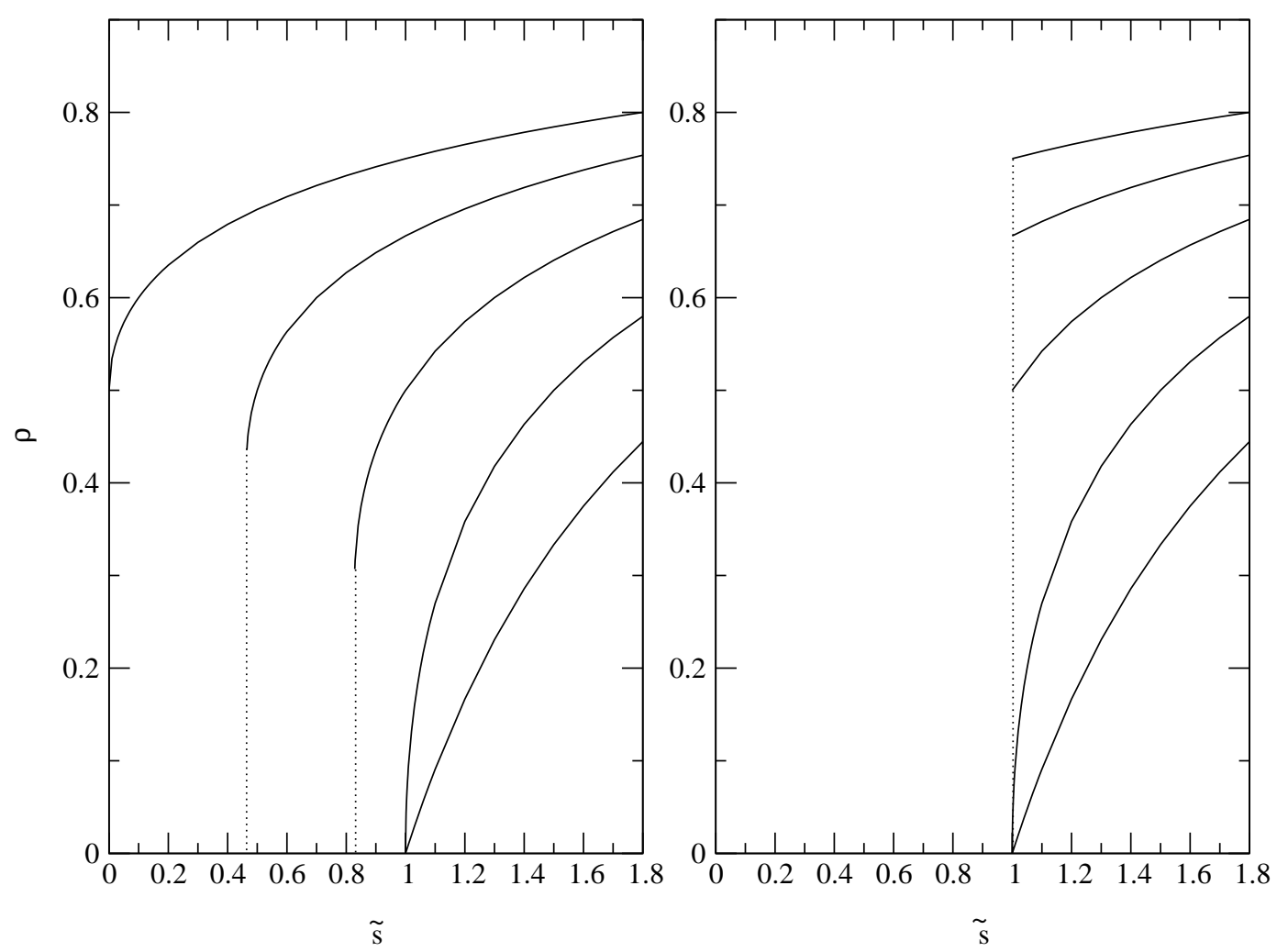

Figure 1: Mean-field steady-state concentration of active sites as a function of the scaled noncatalized self-replication ratio for (bottom to top) $\tilde{c}=$ $0,1,2,3$ and 4 . The initial concentrations are (a) $\rho_{0}=1$ and (b) $\rho_{0}=.001$.

The continuous transition ends at the tricritical point $\tilde{s}_{t}=\tilde{c}_{t}=1$ so that for $\tilde{c}>\tilde{c}_{t}$ the transition between phases $(E)$ and $(E A)$, that takes place at $\tilde{s}=2 \sqrt{\tilde{c}}-\tilde{c}$, as well as the transition between phases $(E A)$ and $(A)$, that occurs at $\tilde{s}=1$, are discontinuous. In particular, the jumps of the densities of replicators are

$$
\Delta \rho=1-1 / \tilde{c}^{1 / 2} \quad \tilde{c}>1
$$

at the transition $(E)-(E A)$, and

$$
\Delta \rho=1-1 / \tilde{c} \quad \tilde{c}>1
$$

at the transition $(E A)-(A)$. These results are conveniently summarized in the phase diagram shown in Fig. 2. It is interesting to note that setting $\tilde{s}=\tilde{c}$ we find, close to the tricritical point,

$$
\rho \approx(\tilde{s}-1)^{1 / 2}
$$

so that the tricritical point is not in the same universality class as the transition observed in the absence of catalytically assisted replication.

The interpretation of our results within the prebiotic evolution context leads to the conclusion that for finite values of $\tilde{c}$ an obligatory sexual replicator cannot 


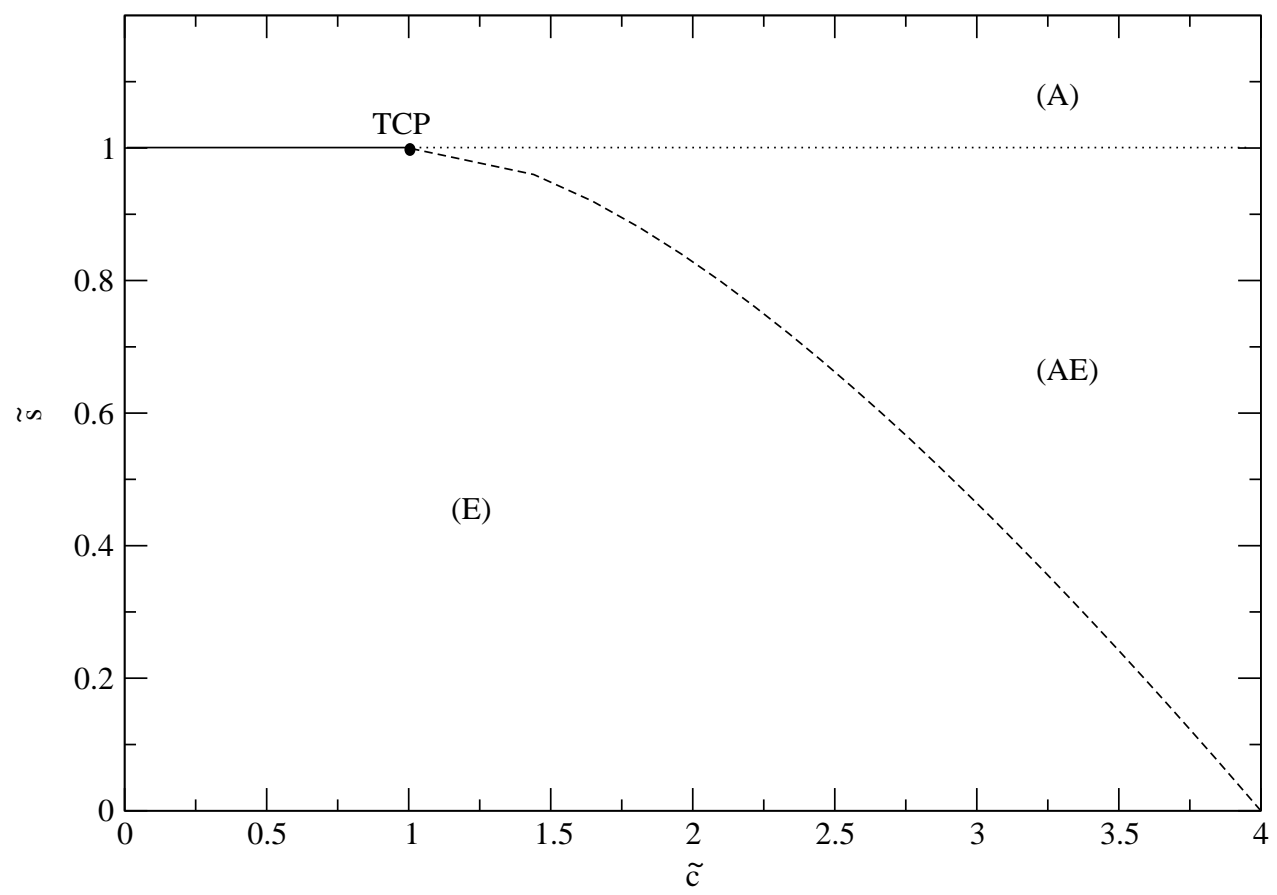

Figure 2: Mean-field phase diagram in the plane $(\tilde{c}, \tilde{s})$ showing the regions of stability of the different steady-state solutions. The continuous transition ending at the tricritical point (TCP) is represented as a solid line and the broken lines indicate discontinuous phase transitions. 
emerge spontaneously (i.e., appear at vanishingly small concentrations). For instance, for $\tilde{s}=0$, the minimal initial density of replicators necessary to engender a prosperous population is $\rho=1 / 2$ at the transition point $\tilde{c}=4$, vanishing as $1 / \tilde{c}$ for large $\tilde{c}$. Actually, an initial colony of replicators is certain to grow from vanishingly small concentrations provided that $\tilde{s}>1$. These conclusions, however, must be taken with caution since in the deterministic limit a vanishingly small concentration means an infinite population of replicators, while one would expect the first replicators to show up as a single or a few copies at most. Of course, a proper understanding of this emergence phenomenon calls for a stochastic approach, which is the subject of the next section.

\section{The position-fixed limit}

The primary aim of this section is to determine what features of the rich phasediagram obtained in the mean-field limit show up also in the opposite limit, where the replicators are fixed on the lattice cells. This is a rather challenging enterprise as, at least in the case of equilibrium phase-transitions, there is no totally unambiguous way by which one can detect the order of the transition through the analysis of finite systems alone [18]. Nevertheless, we tackle this problem using both a steady-state approach for finite lattices and the dynamical Monte Carlo method for lattices of effectively infinite size.

First we measure de density $\rho$ of replicators in the steady state. Our results for two system sizes $(L=100$ and $L=200)$ are shown in Figs. 3 and 4 . For each set of the control parameters we made runs of $10^{5}$ generations, neglecting the first $210^{4}$ generations and recording $\rho$ at steps of 200 generations. A generation corresponds to the simultaneous update of all lattice cells. Each data point is the arithmetic mean of these recorded data. Provided that the population is not extinct, the results are independent of the choice of the initial configuration. In particular, we have made runs starting from all cells occupied or from a seed of only four clustered occupied cells. Furthermore, as in the mean-field limit we have verified that our results depend only on the ratios $s / \gamma$ and $c / \gamma$ so throughout the remaining of this paper the value of the decay constant is held fixed at $\gamma=0.05$. These results clearly indicate the existence of a phase transition separating the active $(\rho>0)$ and empty $(\rho=0)$ phases of the model. Assuming that in the neighborhood of the transition points the density of replicators goes to zero as $\rho \sim\left(\tilde{s}-\tilde{s}_{c}\right)^{\beta}$ and using the least-square method we can estimate both the critical replication rate $\tilde{s}_{c}$ and the critical exponent $\beta>0$. These estimates are presented in table 1 and the quality of the fitting can be appreciated from Fig. 4. The statistical errors are of order of $10^{-3}$ but the systematic errors, which are due mainly to the difficulty to carry out long runs very close to the transition point, are probably much larger. We note that our estimates of $\beta$ for small values of $\tilde{c}$ indicate that in this regime the replicator model belongs to the so-called $(2+1)$ directed-percolation universality class for which $\beta=0.59 \pm 0.02[19]$. It should be pointed out that in finite systems the active regime is a meta-stable one as there is always a finite probability that 


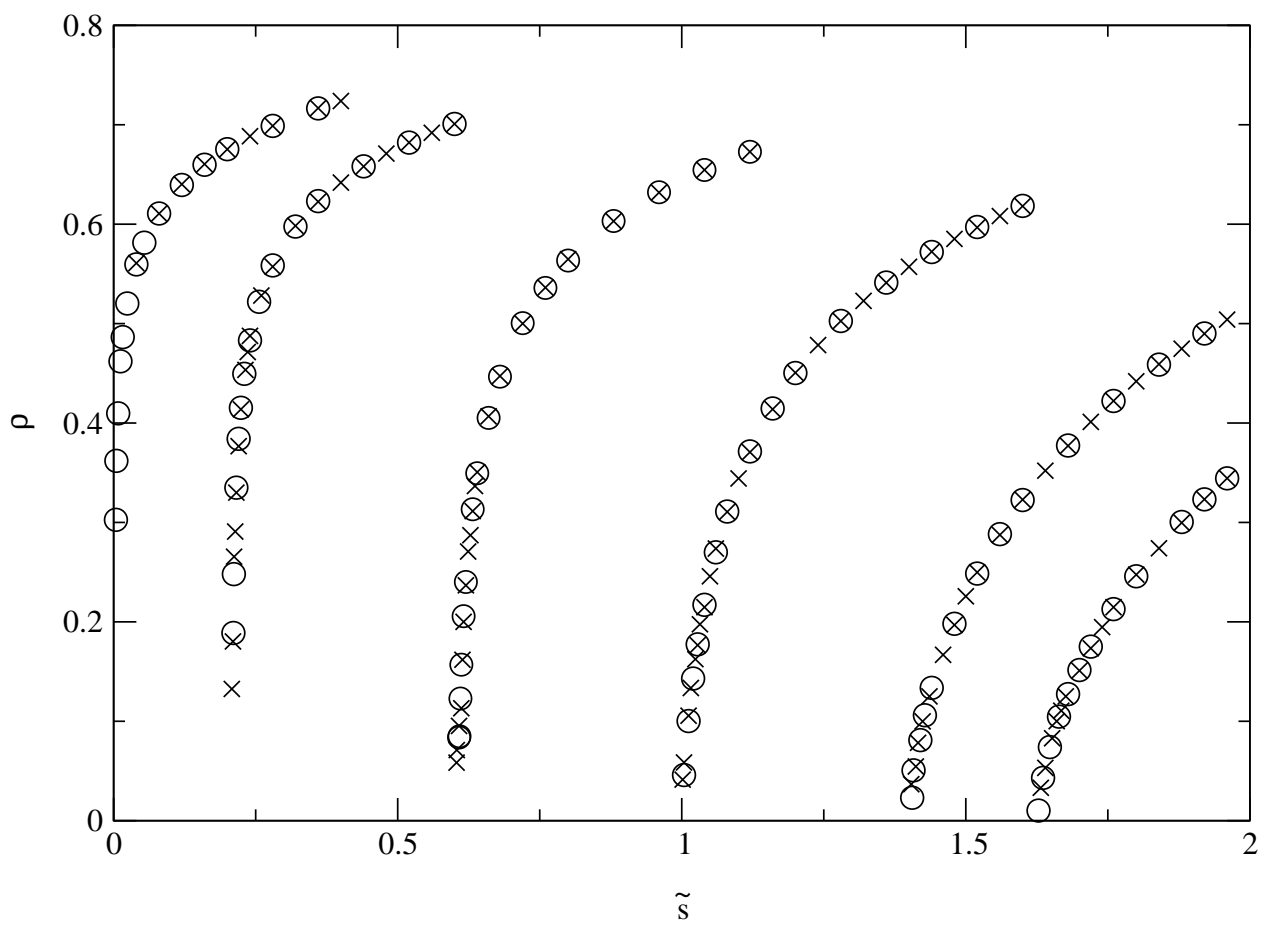

Figure 3: Average density of replicators $\rho$ as a function of $\tilde{s}$ for (left to right) $\tilde{c}=5.70,5.13,3.94,2.58,0.99$ and 0 . The lattice sizes are $L=100(\times)$ and $200(\bigcirc)$.

the colony becomes extinct due to fluctuations in the stochastic dynamics. Since this extinction probability increases towards 1 as the critical point is approached, it is very difficult to obtain reliable estimates of $\tilde{s}_{c}$ and $\beta$ by means of numerical simulations in the steady-state regime. Of course we are aware that if the transition happens to become discontinuous at some value of $\tilde{c}>0$ then the assumption of a power-law singularity at the transition point breaks down (actually $\beta=0$ in this case). In fact, the (anomalous) continuous decrease of $\beta$ as $\tilde{c}$ increases (see table (1) is an indication that this might be the case. Our careless use of the power-law assumption is intentional and aims to illustrate the difficulty of detecting the order of a phase transition using results of steady-state simulations: the abrupt variation of $\rho$ at criticality observed in Fig. 3 for certain values of $\tilde{c}$, which might indicate the occurrence of a first-order irreversible transition, can be explained as a continuous transition with a small exponent $\beta$ as well.

We turn now to the analysis of the spreading behavior of a small colony of replicators settled initially in the center of an otherwise empty lattice of infinite size. More pointedly, the initial colony is composed of four replicators located in the 


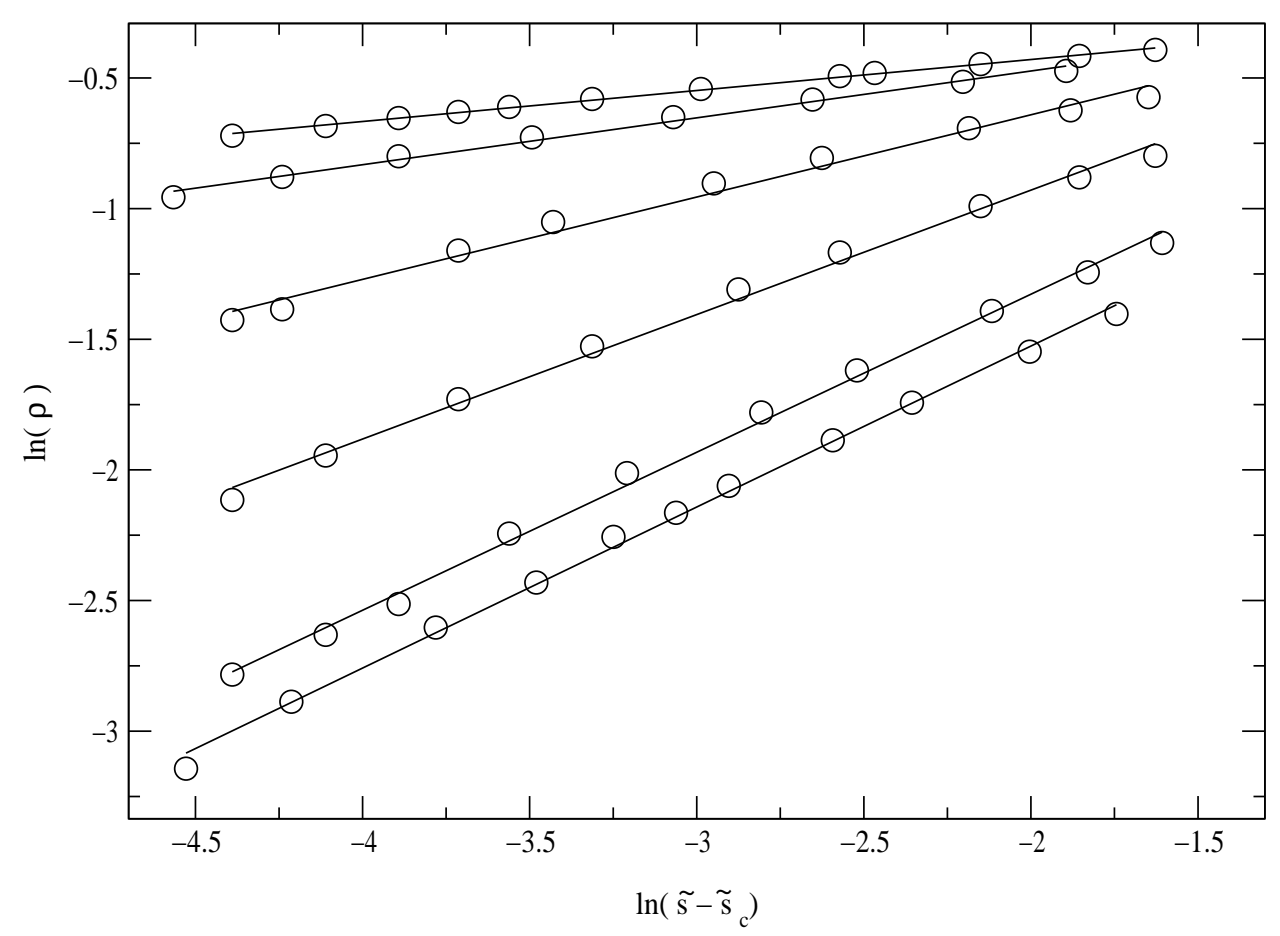

Figure 4: Logarithm plot of the average density $\rho$ as a function of $\left(\tilde{s}-\tilde{s}_{c}\right)$ for (top to bottom) $\tilde{c}=5.70,5.13,3.94,2.58,0.99$ and 0. The values of $\tilde{s}_{c}=\tilde{s}_{c}(c)$ for the different choices of $\tilde{c}$ are given in table 1 and the straight lines are the numerical fitting obtained with those data. Only the data for $L=200$ are presented.

\begin{tabular}{ccc}
\hline$\tilde{c}$ & $\tilde{s}_{c}$ & $\beta$ \\
\hline 0 & 1.625 & 0.61 \\
0.992 & 1.400 & 0.60 \\
2.576 & 1.004 & 0.47 \\
3.936 & 0.608 & 0.30 \\
5.128 & 0.210 & 0.18 \\
5.7008 & 0.004 & 0.12 \\
\hline
\end{tabular}

Table 1: Estimates of the critical point $\tilde{s}_{c}$ and the critical exponent $\beta$ assuming the power-law singularity $\rho \sim\left(\tilde{s}-\tilde{s}_{c}\right)^{\beta}$. 
von Neumann neighborhod of the central empty cell. Finite size effects are absent because the lattice size is taken large enough so that during the time we follow the evolution of the colony the replicators can never reach the lattice boundaries. This of course sets an upper limit to the number of generations we can follow the colony and so, in particular, we let the population evolve up to typically $t=10^{4}$. As usual, we concentrate on the time dependence of the following key quantities [12]: (i) the average number of replicators $n(t)$; (ii) the survival probability of the colony $P(t)$; and (iii) the average mean-square distance over which the replicators have spread $R^{2}(t)$. For each time $t$ we carry out $M=210^{5}$ independent runs, all starting with the same initial colony. Hence $P(t)$ is simply the fraction of runs for which there is at least one replicator in the lattice at time $t$. Since $n(t)$ is an average taken over all runs including those which have already been extinct at generation $t$, the average number of replicators per surviving run is given by the ratio $N(t)=n(t) / P(t)$. Furthermore, noting that $R^{2}(t)$ is averaged only over the surviving runs, we can define the fractal dimension $d_{f}$ of the surviving colonies of replicators at a given time $t$ as $N \sim R^{d_{f}}$.

At the transition points we expect that the measured quantities obey the following scaling laws 12

$$
\begin{aligned}
n(t) & \sim t^{\eta} \\
P(t) & \sim t^{-\delta} \\
R^{2}(t) & \sim t^{z}
\end{aligned}
$$

where $\delta, \eta$ and $z$ are dynamic exponents which are related with the fractal dimension of the clusters of replicators through the equation

$$
d_{f}=2 \frac{\eta+\delta}{z}
$$

In principle, these scaling laws are valid for continuous as well as discontinuous phase transitions, though the scaling relations between the exponents, such as the 'hyperscaling' relation 14.

$$
\frac{1}{2} d z-\eta=2 \delta
$$

where $d$ is the lattice dimension, hold only in the case of continuous transitions.

In Figs. 5 and 6 we present log-log plots of $n(t)$ and $P(t)$, respectively, as functions of $t$ in the vicinity of the critical point for $\tilde{c}=0$. The dependence of $R^{2}(t)$ on $t$ is not shown since, near criticality, the curves for different values of $\tilde{s}$ are clustered together and do not reveal any qualitatively relevant information on the colony evolution. The asymptotic straight lines observed in these figures are the signature of critical behavior while upward and downward deviations indicate supercritical and subcritical behaviors, respectively. A precise estimate for the critical exponents is obtained by considering the local slopes of the curves shown in the previous figures. For instance, the local slope $\delta(t)$ is defined by 14,20

$$
-\delta(t)=\frac{\ln [P(t) / P(t / 5)]}{\ln 5},
$$




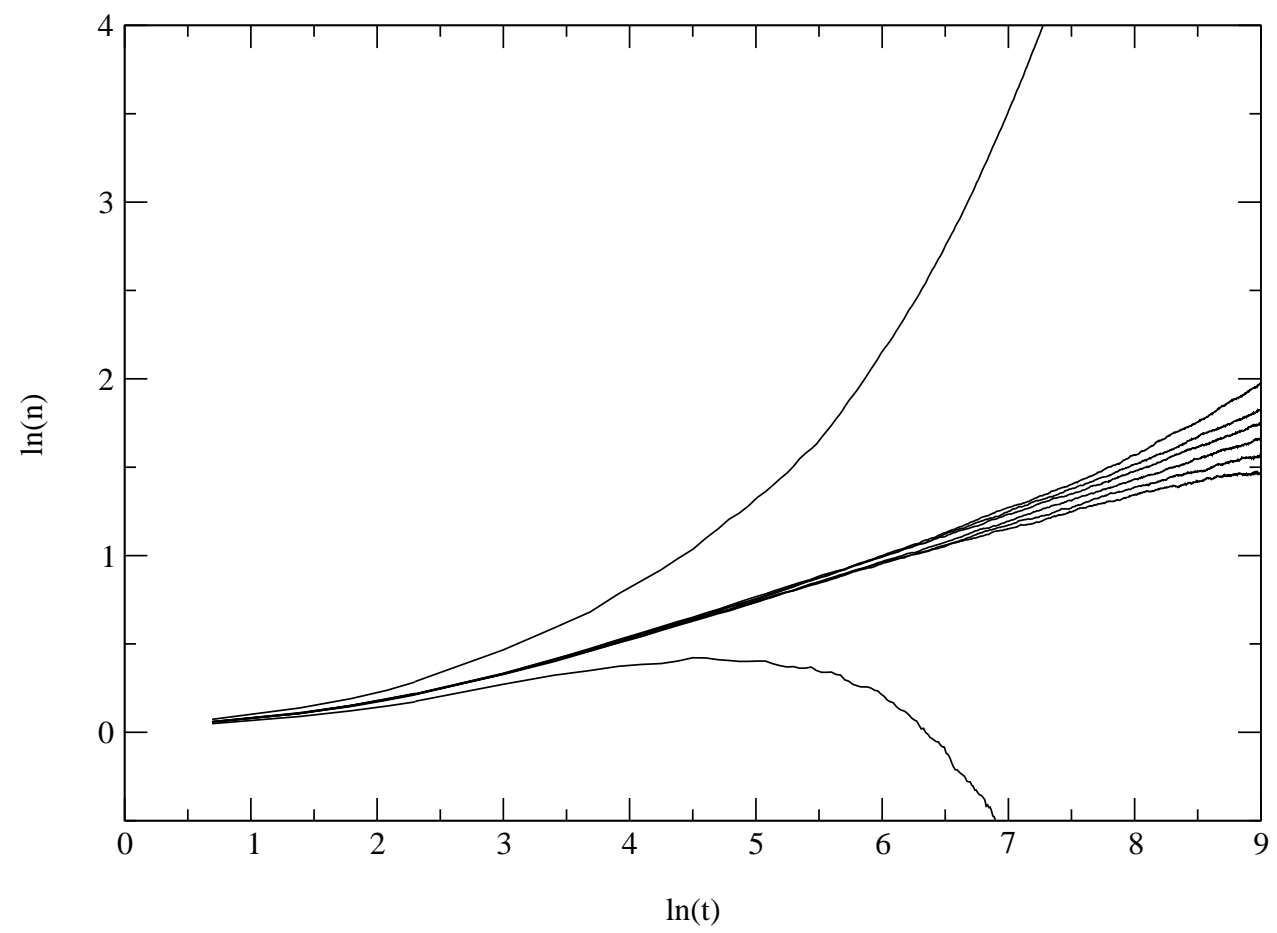

Figure 5: The log-log plot of $n(t)$ as a function of $t$ for $\tilde{c}=0$ and (top to bottom) $\tilde{s}=1.80,1.632,1.630,1.629,1.628,1.627,1.626$, and 1.52.

which for large $t$ behaves as

$$
\delta(t) \sim \delta+\frac{a}{t}
$$

where $a$ is a constant. Analogous expressions hold for $\eta(t)$ and $z(t)$. Hence plots of the local slopes as functions of $1 / t$ allow the calculation of the critical exponents. Using this procedure we find $\tilde{s}_{c}=1.628 \pm 0.001$ which yields the exponents $\eta=$ $0.23 \pm 0.01, \delta=0.45 \pm 0.01$ and $z=1.13 \pm 0.01$. The error in $\tilde{s}_{c}$ is estimated by determining two values of $\tilde{s}$ as close as possible to the critical point for which upward and downward deviations can be observed, while the errors in the critical exponents are, as usual, the statistical errors obtained by fitting the local slopes by straigh lines in the large $t$ regime. Our exponents are in good agreement with those of the $(2+1)$ directed-percolation [14] and satisfy very well the hyperscaling relation (16) thus indicating that the transition in the limit $\tilde{c}=0$ is continuous, as expected.

The results of the spreading analysis for the other extreme case, $\tilde{s}=0$, which models a population of obligatory sexual replicators are shown in Figs. 7 and 8 . Although the dependence of $\ln P(t)$ on $t$ is similar to that observed in the previous case, the behavior pattern of $\ln n(t)$ (see Fig. (7) is rather different: in the 


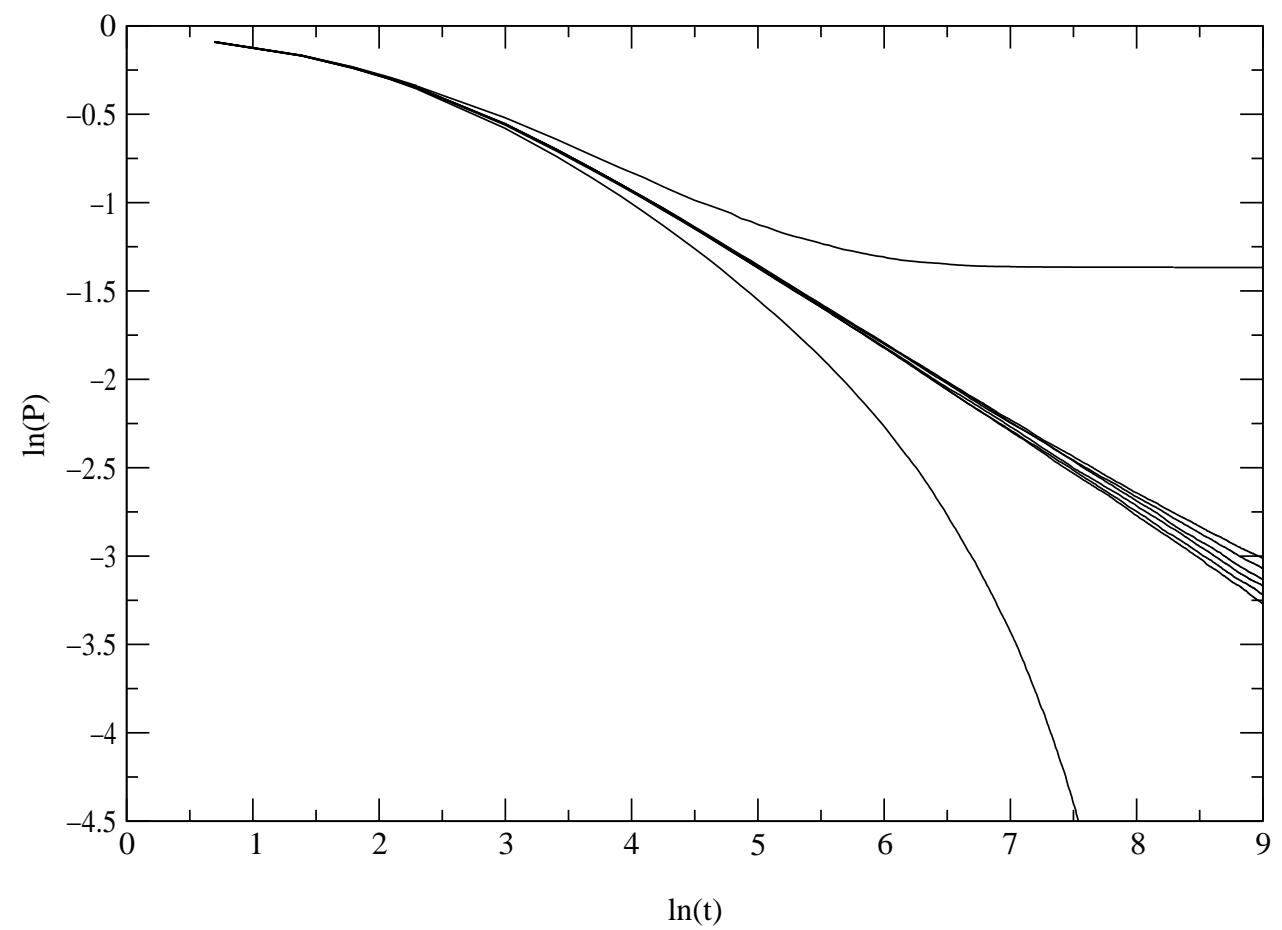

Figure 6: Same as fig. 5 but for $P(t)$. 
supercritical regime (i.e., $\left.\tilde{c}>\tilde{c}_{c}\right) n(t)$ first increases reaching a maximum, then decreases reaching a minimum and finally starts to increase monotonically again. This pattern is illustrated better in Fig. 9, which shows the spreading results for $\tilde{s}=0.2$. Analysis of these figures, which exceptionally show the colony evolution up to $210^{4}$ generations, suggests that a flat line separates the supercritical and the subcritical regimes implying thus the vanishing of the exponent $\eta$ at the transition point. Furthermore, the qualitatively distinct behavior patterns of $\ln n(t)$ illustrated in Figs. 5, 7 and 9 can be used to identify unambiguously the order of the nonequilibrium phase transition and hence to estimate the location of the tricritical point. To appreciate how the time dependence of $\ln n(t)$ in the supercritical regime changes continuously from the simple monotonic increase for $\tilde{s}=0$ to the complicated behavior described above for $\tilde{c}=0$ we present in Figs. 10 and 11 $\log -\log$ plots of $n(t)$ as a function of $t$ for $\tilde{s}=0.6$ and $\tilde{s}=1.0$, respectively. In fact, analysis of Figs. 7 to 11 suggests that the turning point between those distinct behavior patterns occurs when the maximum and the minimum of $n(t)$ coincide, i.e., the critical curve $\ln n(t)$ vs. $\ln t$ has an inflection point (see Fig. 10). The value of $\tilde{s}$ and the corresponding $\tilde{c}_{c}$ at which this behavior occurs are then identified as the coordinates of the tricritical point. Applying this procedure we find $\tilde{s}_{t}=0.60 \pm 0.04$ and $\tilde{c}_{t}=4.00 \pm 0.20$.

The transition points and the dynamic exponents obtained via the scaling laws (12)-(14) and via the analysis of the local slopes are summarized in table 2. The errors in the estimates of the transition points are calculated as described before for the case $\tilde{c}=0$. Except for that case, we refrain from presenting the (statistical) errors in the exponents since the systematic errors are unusually large, due probably to the crossover behavior among (at least) three different universality classes. For instance, in the vicinity of the transition point for $\tilde{s}=0.6$, analysis of the local slope $\delta(t)$ up to $t=500$ indicates a clear tendency to the asymptotic value $\delta \approx 1$ while for $t>500$ the tendency suddenly changes towards the asymptotic value $\delta \approx 0.6$. A similar phenomenon occurs for $\tilde{s}=1.0$ also: the initial tendency is towards $\delta \approx 0.6$ and then changes towards $\delta \approx 0.45$ for larger times. As a result the estimate of the exponents becomes strongly dependent on the precise location of the transition points, which requires even better statistics as well as much longer runs. We leave this interesting research line which includes, for instance, the identification of the universality class of the tricritical point to a future, more technical contribution.

The evidences in support of our claim that, similarly to the mean-field limit, in the position-fixed limit the nonequilibrium phase transition between the empty and active phases is discontinuous for small $\tilde{s}$ and so there is a tricritical point in the phase diagram of the model are threefold: First, the vanishing of the exponent $\eta$ in this range signalizes a distinct asymptotic behavior of the average number of replicators $n(t)$. Second, the hyperscaling relation (16) is clearly violated for small $\tilde{s}$ while it is satisfied in the regime where we expect the transition to be a secondorder one. Third, using Eq. (15) and the data of table 2 we find $d_{f} \approx d=2$ in the region of small $\tilde{s}$ indicating that the clusters of replicators are not fractal objects, in contrast to the clusters observed in the vicinity of a second-order transition (we find, for instance, $d_{f} \approx 1.21$ for $\tilde{c}=0$ ). This point is illustrated in Figs. 12 and 13 


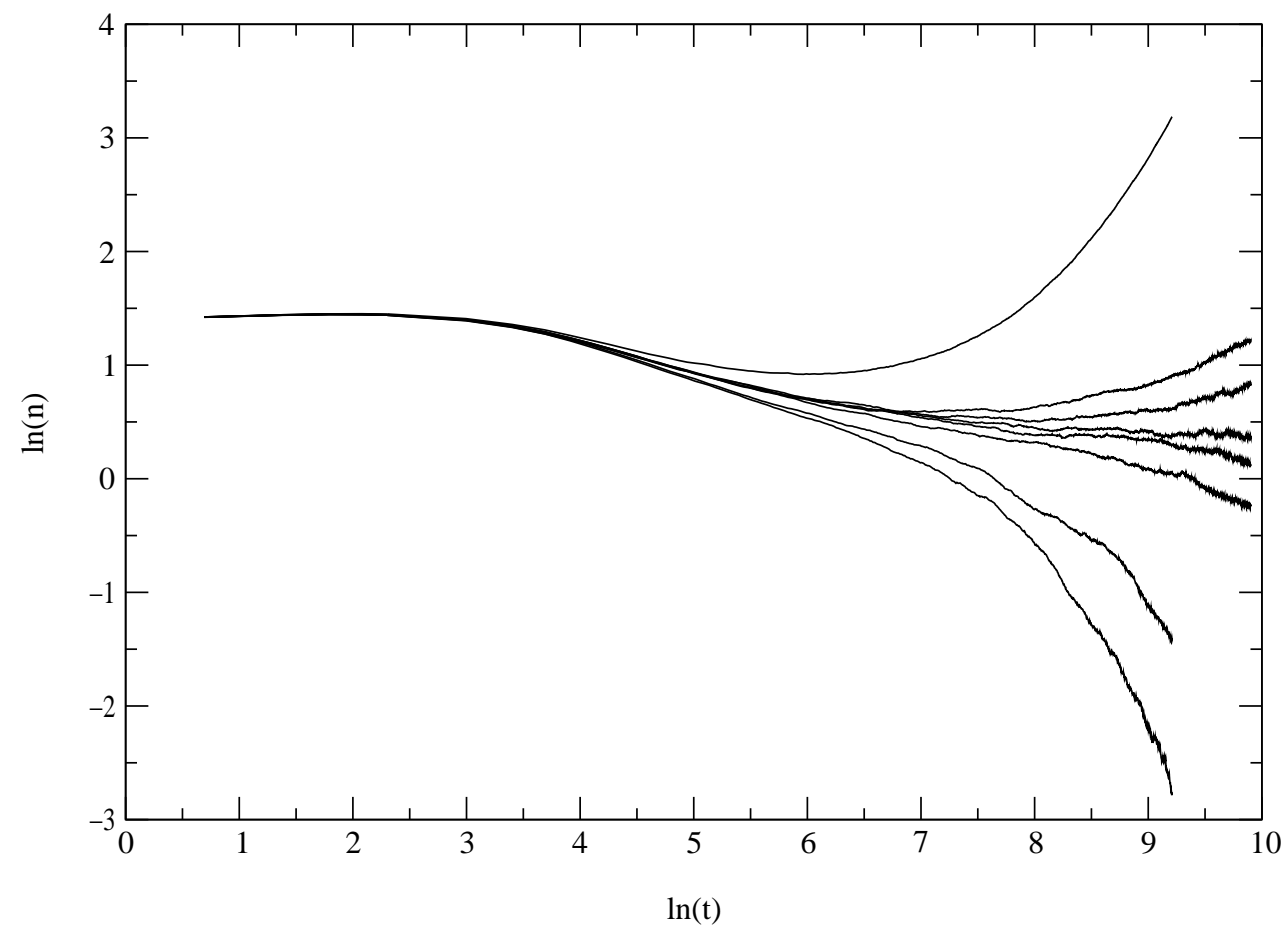

Figure 7: The log-log plot of $n(t)$ as a function of $t$ for $\tilde{s}=0$ and (top to bottom) $\tilde{c}=5.760,5.712,5.709,5.704,5.702,5.701,5.680$ and 5.664 .

\begin{tabular}{ccccc}
\hline$\tilde{s}$ & $\tilde{c}_{c}$ & $\eta$ & $\delta$ & $\mathrm{z}$ \\
\hline 0 & $5.704 \pm 0.005$ & -0.03 & 0.96 & 0.98 \\
0.2 & $5.152 \pm 0.008$ & -0.004 & 0.80 & 1.03 \\
0.6 & $3.952 \pm 0.008$ & 0.14 & 0.63 & 1.08 \\
0.8 & $3.296 \pm 0.008$ & 0.22 & 0.54 & 1.11 \\
1.0 & $2.584 \pm 0.008$ & 0.20 & 0.51 & 1.11 \\
1.2 & $1.832 \pm 0.008$ & 0.25 & 0.49 & 1.13 \\
1.4 & $1.008 \pm 0.008$ & 0.23 & 0.47 & 1.11 \\
$1.628 \pm 0.001$ & 0 & $0.23 \pm 0.01$ & $0.45 \pm 0.01$ & $1.13 \pm 0.01$ \\
\hline
\end{tabular}

Table 2: Critical dynamic exponents calculated from the slopes of the straight lines at the transition points $c_{c}(s)$. 


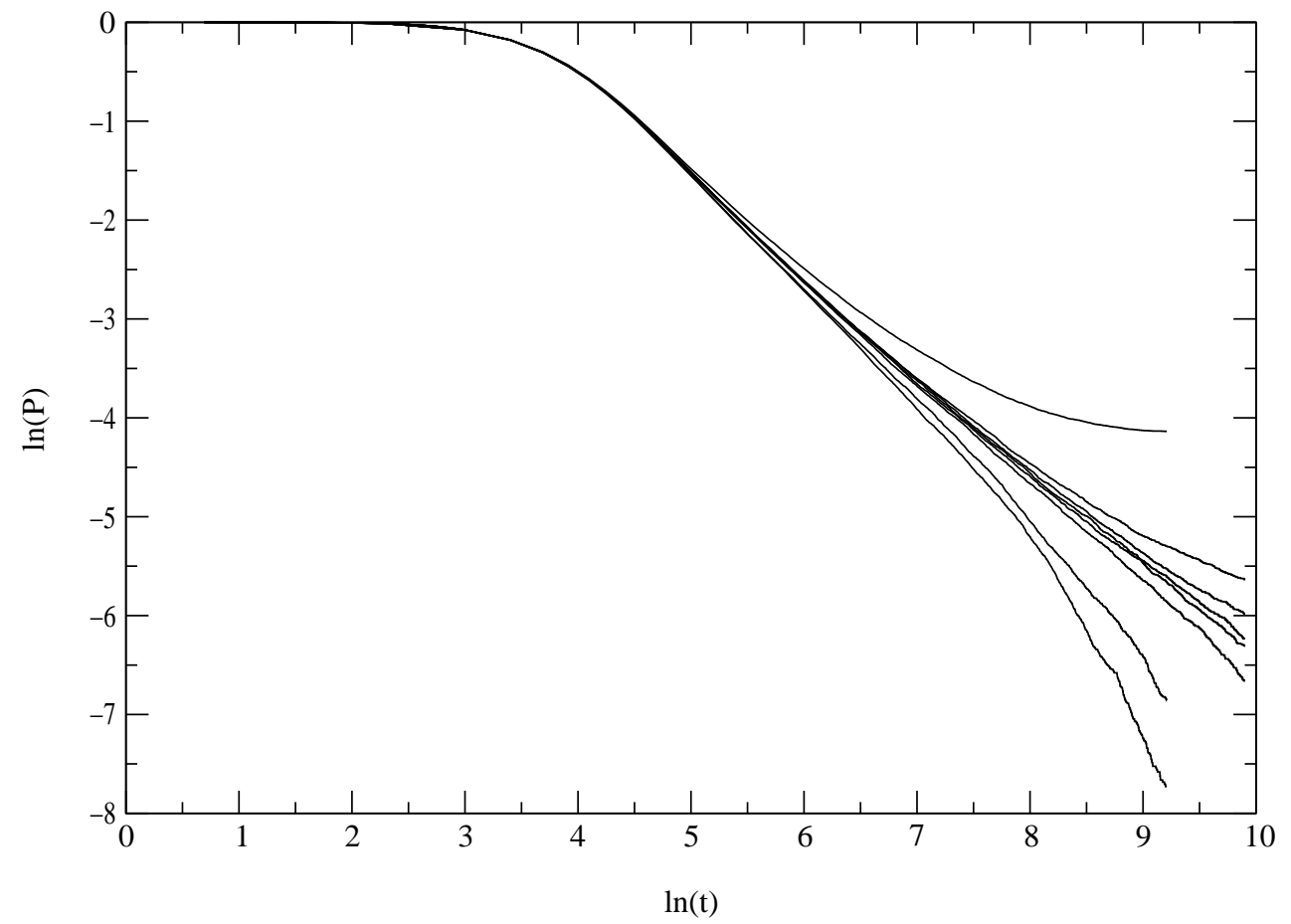

Figure 8: Same as fig. 7 but for $P(t)$. 


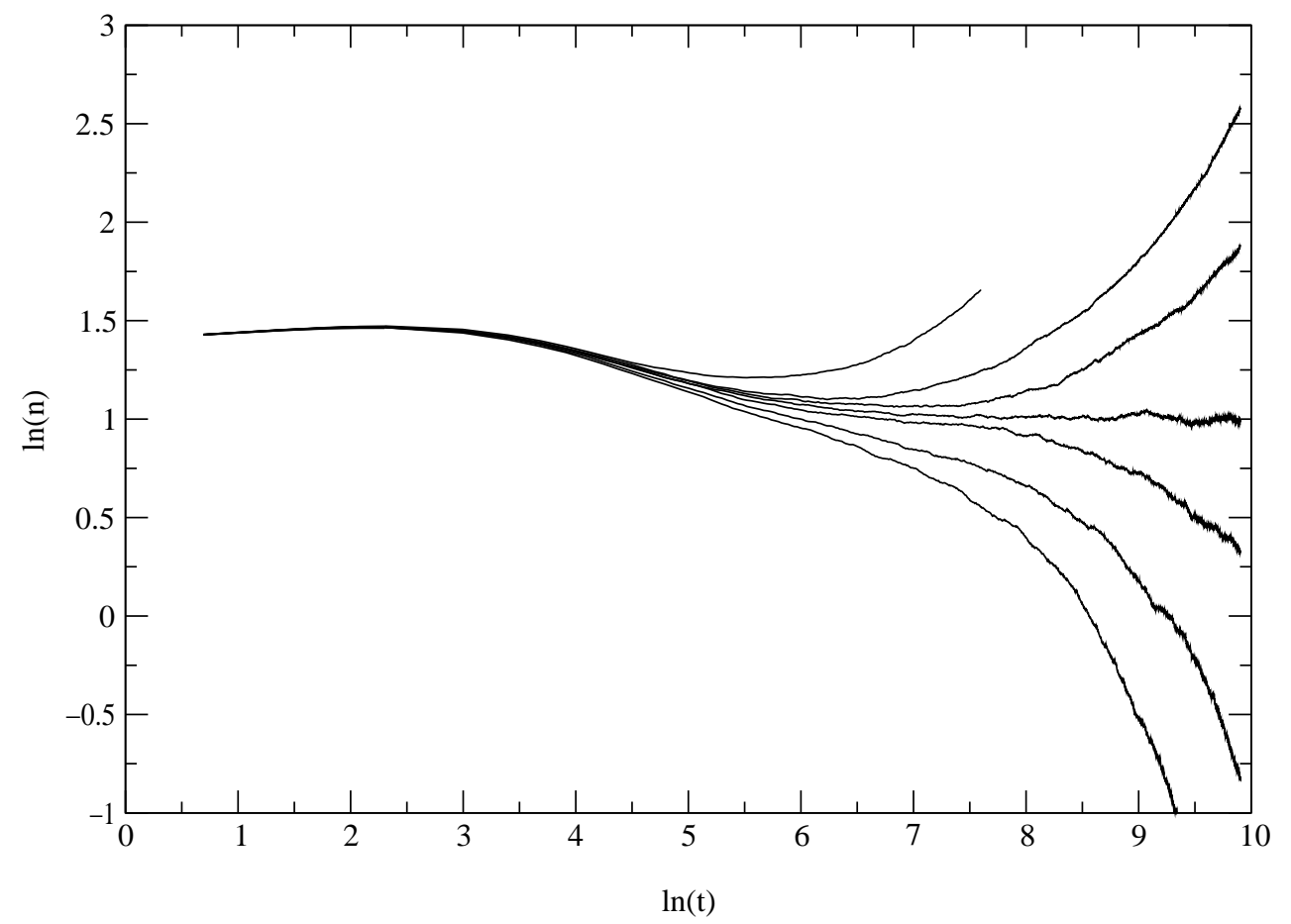

Figure 9: The log-log plot of $n(t)$ as a function of $t$ for $\tilde{s}=0.2$ and (top to bottom) $\tilde{c}=5.20,5.168,5.160,5.152,5.144,5.136$ and 5.120 . 


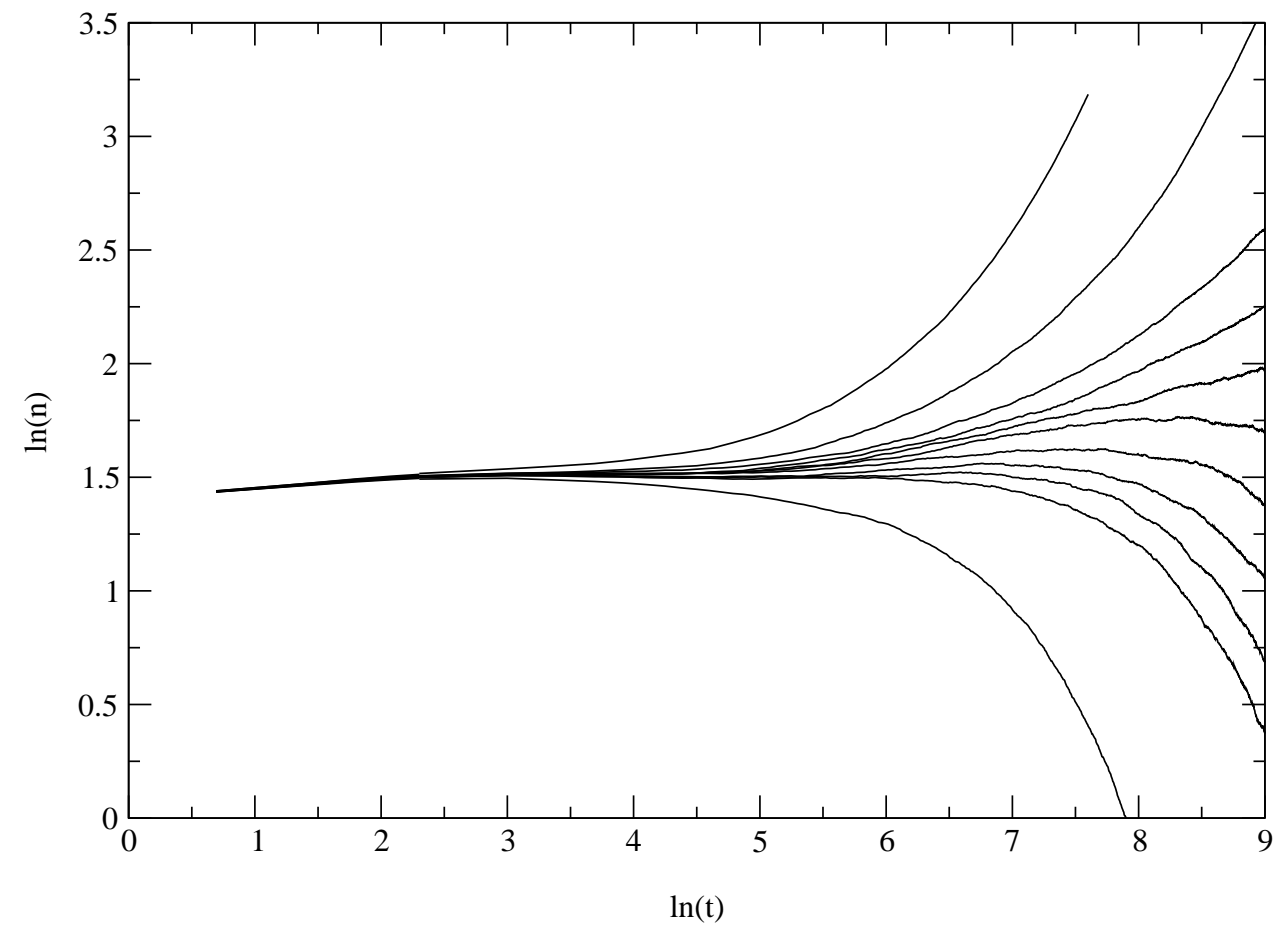

Figure 10: The log-log plot of $n(t)$ as a function of $t$ for $\tilde{s}=0.6$ and (top to bottom) $\tilde{c}=4.080,4.000,3.968,3.960,3.952,3.944,3.936,3.928,3.920,3.912$ and 3.840 . 


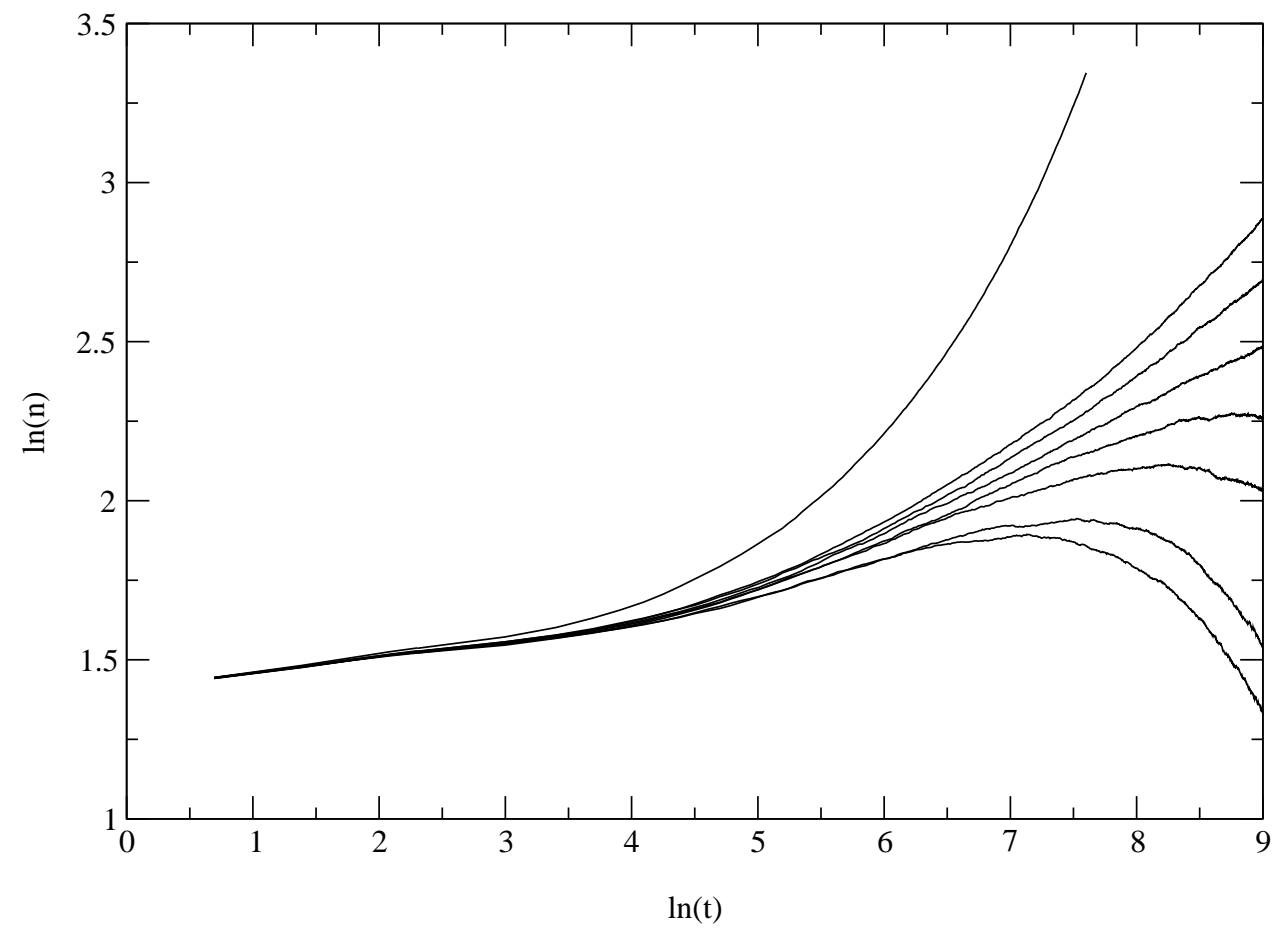

Figure 11: The log-log plot of $n(t)$ as a function of $t$ for $\tilde{s}=1.0$ and (top to bottom) $\tilde{c}=2.720,2.600,2.592,2.584,2.576,2.568,2.552$ and 2.544 . 


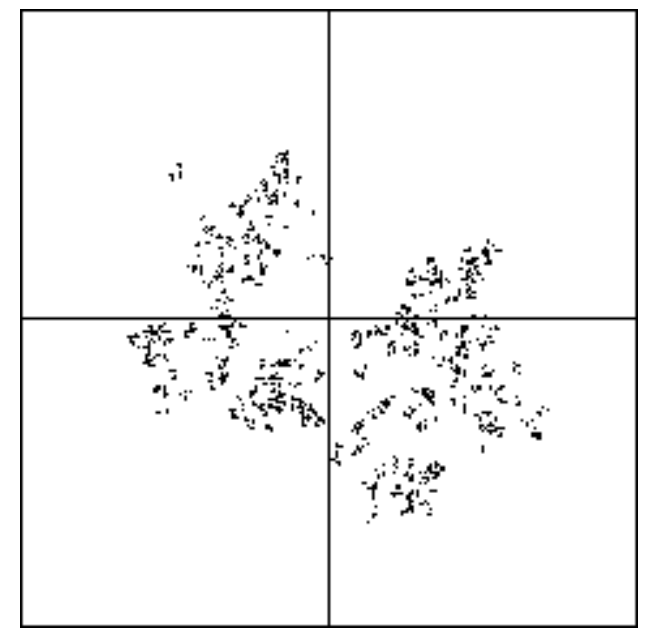

Figure 12: Snapshot of the lattice configuration at $t=10^{4}$ showing the colony of replicators (dots). The parameters are $\tilde{c}=0$ and $\tilde{s}=1.69$. The initial colony of four replicators was placed in the middle of the $200 \times 200$ lattice.

which show snapshots of typical colonies at $t=10^{4}$ for the two extreme cases. In both figures the relative distances to the critical points are the same. The reason for the colonies of obligatory replicators to be much denser than those of malthusian replicators is that the number of replicators per surviving colony $\left(N(t) \sim t^{\eta+\delta}\right)$ increases roughly as $t$ for the former and as $t^{0.7}$ for the latter. In addition, the average square distance over which the initial colony has spread from the center of the lattice at time $t$ is roughly of the same order in both extremes as indicated by the values of the exponent $z$.

Finally, in Fig. 14 we present the phase diagram for the position-fixed limit. In this case there are only two phases, namely, the empty phase $(E)$ characterized by a vanishing probability of survival $P_{\infty} \equiv \lim _{t \rightarrow \infty} P(t)=0$ and the phase $(E A)$ where the active and empty states can occur with probabilities $P_{\infty}$ and $1-P_{\infty}$ (see Figs. 6 and 8), respectively. We note that the population size is effectively unlimited so that extinction is not certain to occur as in the steady-state analysis of finite systems. It is worth emphasizing that for finite production rates one has $P_{\infty}<1$ and so there is always a nonvanishing probability of extinction. (Actually, 


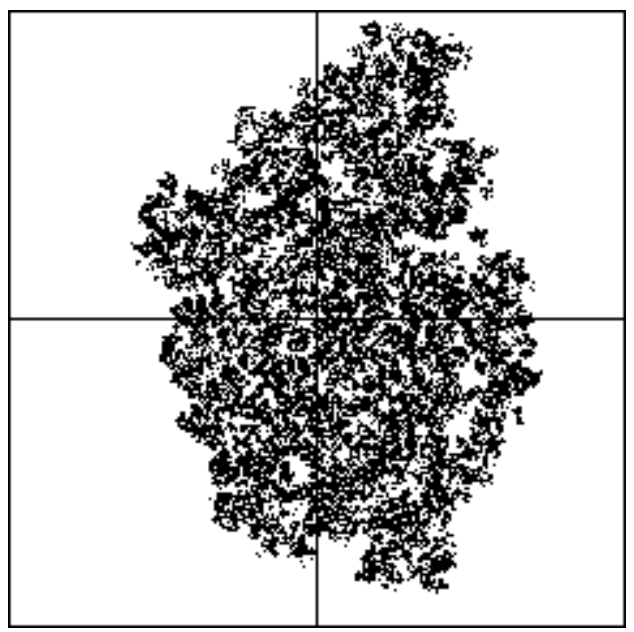

Figure 13: Same as Fig. 12 but for $\tilde{s}=0$ and $\tilde{c}=5.92$. 


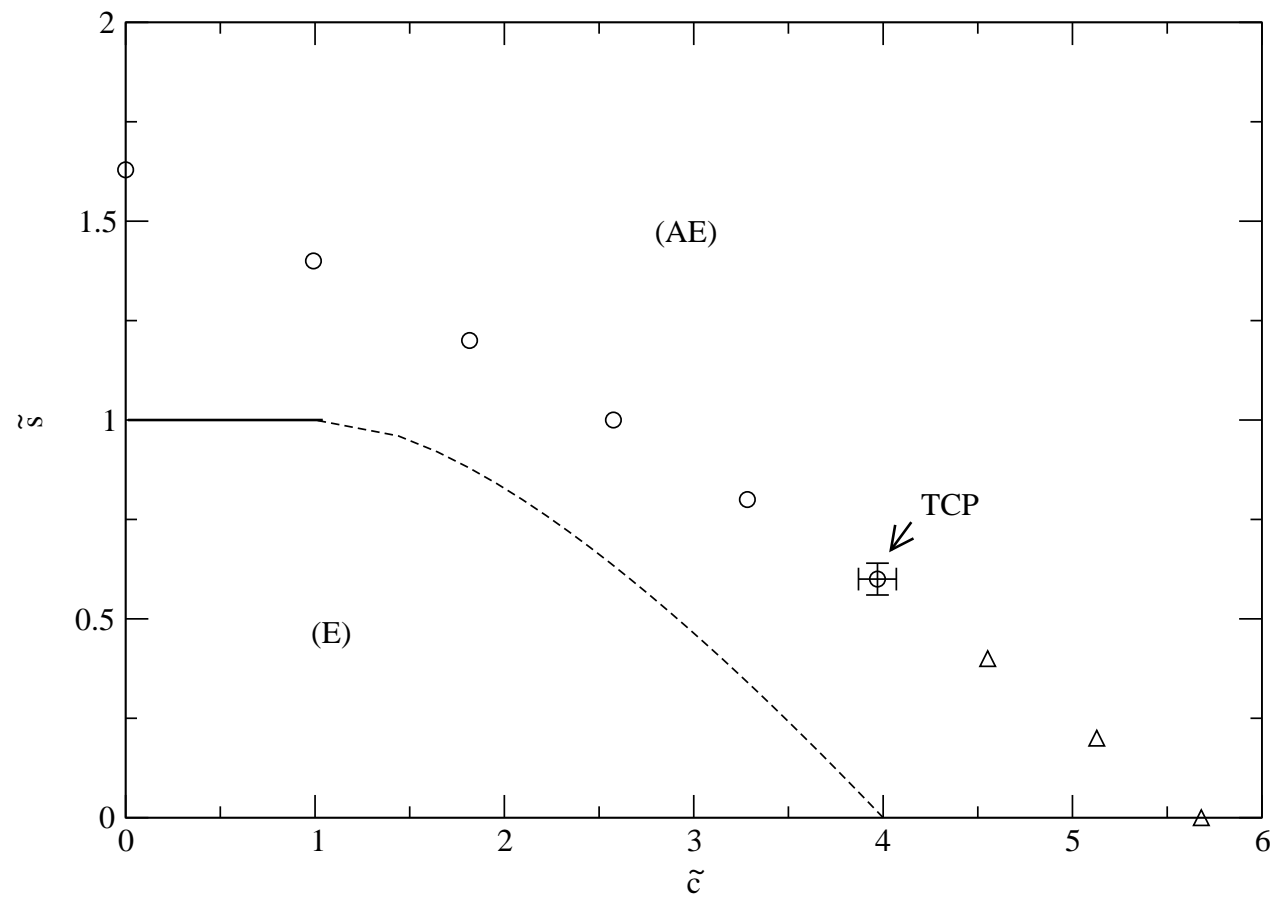

Figure 14: Phase diagram in the plane $(\tilde{c}, \tilde{s})$ for the position-fixed limit. The continuous and discontinuous transition points are represented by the symbols $\bigcirc$ and $\triangle$, respectively. The error bars represent the uncertainty in the location of the tricritical point (TCP) while for the other data points the error bars are smaller than the symbol sizes. For sake of comparison the mean-field phase-diagram (solid and broken lines) is also presented.

$P_{\infty}$ depends on the number and location of the replicators in the initial colony, but the conditions we have chosen are the most relevant for the emergence of life problem.) This result contrasts to our findings in the mean-field limit that the fixed point associated to the empty phase becomes unstable for $\tilde{s}>1$ so that even starting with a vanishingly small concentration of replicators the population never dies out. As mentioned before, the reason for this discrepancy is not the difference in mobility of the replicators but the fact that the mean-field analysis actually considers an infinite population and hence it fails to take into account the stochastic fluctuations that could drive a small population to extinction.

For sake of completeness, we should mention that we have also carried out a similar analysis for one-dimensional lattices (chains). While the results for the mean-field limit are of course the same (provided we properly redefine the dimensionless parameters $\tilde{c}$ and $\tilde{s}$ ), the fixed-point limit has some distinct features that are worth-mentioning. In particular, we find no evidence for a first-order transition; instead, we find that in both extremes $\tilde{c}=0$ and $\tilde{s}=0$ the empty and active phases are separated by a second-order phase transition that belongs to the $(1+1)$ directed-percolation universality class [12]. Interestingly, in one-dimension 
the steady-state analysis of finite chains is rendered practically useless by the very pronounced finite-size effects, which are probably due to the proximity to the lower critical dimension of the model.

\section{Conclusion}

In this paper we have focussed on the prior step in the evolution of life: What are the necessary conditions for small colonies of molecules capable of making copies of themselves via some template mechanism to persist? This step must be passed before one can consider issues such as the outcome of the competition between the replicators and their defective copies [1, 23] or between different kinds of replicators [2. 5]. As a model of replicator we have considered the well-established hypercyclic replicator (one-membered hypercycle) which incorporates two independent mechanisms of replication, namely, the direct template replication reaction (1i) and the catalytically-assisted template replication reaction (3), whose rates are proportional to the parameters $s$ and $c$, respectively. Furthermore, motivated by the modern theories for the evolution of life that suggest a scenario of diffusion-controlled chemical reactions taking place on adsorbing surfaces (probably pyrite) where each reactant can move randomly on the surface [16, 17], we have considered a two-dimensional lattice model where each reactant can occupy one of the lattice cells.

Since the diffusion process of reactants complicates considerably the analysis, we have focussed on the two extreme situations: the infinite diffusion or meanfield limit and the position-fixed or contact process limit. The expectation is that features common to both limits should also be present in the more realistic, intermediate situations. In both cases we found rich phase diagrams showing the regions in the plane $(c, s)$ where the replicators persist (active phase) and die out (empty phase): these regions are separated by second-order nonequilibrium phase transitions which turn into first-order ones at tricritical points. The dynamic Monte Carlo method has proven very well suited to our investigation of the position-fixed limit not only because the method is based on the analysis of the spreading behavior of a small colony of active cells, which is exactly the problem we are interested in, but because, rather surprisingly, it allows an unambiguous identification of the order of the nonequilibrium phase transition. In addition, we show that the continuous transition is in the universality class of the $(2+1)$ directed percolation.

Some remarks on the apparent similarity between Schlögl's first and second models and the replicator models studied in this paper are in order 15. In fact, irreversible versions of Schlögl's first and second models are recovered when the reactant $E$ is eliminated from reactions (11) and (3), respectively, so that the existence of an empty cell containing source materials is not required for replication. Interestingly, this difference is not important in the case of malthusian replicators $(c=0)$ since this model has the same critical behavior as Schlögl's first model, namely, a second-order phase transition which is in the same universality class of the $(d+1)$ directed percolation (the mean-field limit is obtained for $d \geq 4)$ [21]. The comparison between Schlögl's second model and obligatory sexual replicators 
$(s=0)$ is more involved. On the one hand, the mean-field analysis of Schlögl's second model predicts a first-order phase transition [15] but Monte Carlo calculations indicate that the transition for $d<4$ is a second-order one which is actually in the same universality class as the transition in Schlögl's first model [22]. On the other hand, our results for the case $s=0$ show that a directed-percolation-like, secondorder phase transition takes place for $d=1$ only, the transition being discontinuous for higher dimensions $(d \geq 2)$. We should mention, however, that the Monte Carlo implementation of Schlögl's second model actually allows the diffusion of reactants to neighboring cells and, in addition, allows a cell to shelter more than one reactant [22] so that a comparison with the position-fixed limit may not be appropriate. In any event, it is our opinion that the hypercyclic replicator model should not be viewed as a mere variant of Schlögl's models; rather it is a well-established model of chemical evolution [1, 2, 5] which, as far as we know, has not been studied beyond the mean-field limit.

Although the simple replicator model considered in this paper turned out to be a quite exciting model of nonequilibrium phase transitions, we should not lose sight of the original purpose of this work and so, at this stage, it is important to highlight the relevance of our results to the origin of life issue. In fact, the mere existence of a phase transition between the empty and the active regimes poses a difficulty to our scenario of the emergence of life since the scaled production rate $\tilde{s}$ of the spontaneously created self-replicating molecule must be larger than some threshold value already at the outset. Though increasing the mobility of the reactants decreases this threshold somewhat, that scenario would be more plausible if replicators with vanishingly small production rates could also thrive. The situation becomes even worse in the case of first-order transitions: in the deterministic meanfield limit the initial abundance of the spontaneously created replicators should be large as well, while in the stochastic position-fixed limit the probability of survival in the vicinity of the transition point is some orders of magnitude smaller than in the case of a second-order transition (see Figs. 6 and 8). Furthermore, our results indicate that some important conclusions, such as the certainty of survival for $\tilde{s}>1$ or the role played by the initial concentration of replicators near a discontinuous transition, are actually artifacts of the deterministic formalism commonly used to study chemical evolution.

In summary, our results show the necessity of adding some new elements to the standard scenario for the emergence of life whose effect would be to avoid the phase-transitions, allowing thus inefficient replicators to thrive at this first stage of life. Only then one can invoke natural selection and imperfect replication to boost the replication rates. In addition, our results point to the inadequacy of the deterministic mean-field or chemical kinetics formulation to address the origin of life issue and suggest as an alternative stochastic formulation the dynamic Monte Carlo method that has been extensively used in the physics literature to study nonequilibrium phase transitions.

The work of J.F.F. is supported in part by Conselho Nacional de Desenvolvi- 
mento Científico e Tecnológico $(\mathrm{CNPq})$ and Fundação de Amparo à Pesquisa do Estado de São Paulo (FAPESP), Proj. No. 99/09644-9. C.P.F. is supported by FAPESP.

\section{References}

[1] M. Eigen, Naturwissenschaften 58, 465 (1971).

[2] M. Eigen and P. Schuster, Naturwissenchaften 65, 7 (1978).

[3] J. Maynard Smith, Nature 280, 445 (1979).

[4] D. S. Wilson, The Evolution of Populations and Communities (Benjamin/Cumings, Menlo Park, 1980).

[5] M. R. Michod, Amer. Zool. 23, 15 (1983).

[6] E. Szathmáry and J. Maynard Smith, J. Theor. Biol. 187, 555 (1997).

[7] D. Alves, P. R. A. Campos, A. T. C. Silva and J. F. Fontanari, Phys. Rev. E 63, 011911 (2001).

[8] M. C. Boerlijst and P. Hogeweg, Physica D 48, 17 (1991).

[9] M. C. Boerlijst and P. Hogeweg, Physica D 88, 29 (1995).

[10] P. Chacón and J. C. Nuño, Physica D 81, 398 (1995).

[11] M. B. Cronhjort and C. Blomberg, Physica D 101, 289 (1997).

[12] P. Grassberger and A. de La Torre, Ann. Phys. 122, 373 (1979).

[13] P. Grassberger, Math. Biosc. 63, 157 (1983).

[14] P. Grassberger, J. Phys. A 22, 3673 (1989).

[15] F. Schlögl, Z. Phys. 253, 147 (1972).

[16] G. Wächtershäuser, Microb. Rev. 52, 452 (1988)

[17] J. Maynard Smith and E. Szathmáry, The Major Transitions in Evolution (Freeman, Oxford, 1995).

[18] M. S. S. Challa, D. P. Landau, K. Binder, Phys. Rev. B, 34, 1841 (1986).

[19] K. De'Bell and J. W. Essam, J. Phys. A 16, 3553 (1983)

[20] I. Jensen, H. C. Fogedby and R. Dickman, Phys. Rev. A 41, 3411 (1990).

[21] H. K.Janssen, Z. Phys. B 42, 151 (1981)

[22] P. Grassberger, Z. Phys. B 47, 365 (1982)

[23] P. R. A. Campos, J. F. Fontanari and P. F. Stadler, Phys. Rev. E 61, 2996 (2000). 